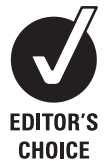

${ }^{1}$ Department of Medicine and Therapeutics, University of Aberdeen, Foresterhill, Aberdeen, UK; ${ }^{2}$ Aberdeen Royal Infirmary, Foresterhill, Aberdeen, UK

\section{Correspondence to:}

Dr C Counsell, Department of Medicine and Therapeutics, University of Aberdeen, Polwarth Building, Foresterhill, Aberdeen AB25 2ZD, UK; carl.counsell@ abdn.ac.uk

Received 16 January 2008 Revised 3 April 2008 Accepted 21 April 2008 Published Online First 9 May 2008

\title{
Changes in diagnosis with follow-up in an incident cohort of patients with parkinsonism
}

\author{
R Caslake, ${ }^{1}$ J N Moore, ${ }^{2}$ J C Gordon, ${ }^{1}$ C E Harris, ${ }^{1}$ C Counsell ${ }^{1}$
}

\begin{abstract}
Background: Accurate diagnosis of the cause of parkinsonism during life can be difficult, particularly at presentation, but few studies have described changes in clinical diagnosis over time and the effect of applying strict research criteria.

Methods: Incident patients with a possible/probable diagnosis of degenerative or vascular parkinsonism had a standardised assessment at diagnosis and at yearly intervals thereafter at which the most likely clinical diagnosis was recorded without strict application of research criteria. Four years after the beginning of the incident period, formal research criteria were applied retrospectively using patient records at baseline and the latest yearly follow-up.
\end{abstract}

Results: Of 82 incident patients, 66 underwent at least 1 year of follow-up. After a median follow-up of 29 months, clinical diagnosis had changed in $22(33 \%)$. Most (82\%) changes occurred in the first year and were due to the development of atypical clinical features, particularly early cognitive impairment; the results of brain imaging; responsiveness to levodopa; and the rate of disease progression. Diagnosis on research criteria differed from latest clinical diagnosis in eight participants (12\%). Research criteria gave a "probable" diagnosis in $71 \%$ of parkinsonian patients at follow-up but in only $15 \%$ at the initial assessment.

Discussion: The clinical diagnosis of the cause of parkinsonism at presentation was often incorrect, even when made by those with a special interest. In particular, Parkinson's disease was overdiagnosed. Research criteria were often unhelpful in clarifying the diagnosis, even after a median of 29 months of follow-up. Further research is required to identify factors that may be used to improve the accuracy of diagnosis at initial assessment.

Parkinson's disease (PD) is a neurodegenerative disorder characterised by tremor, rigidity, bradykinesia and postural instability, and associated with a number of non-motor features, including progressive cognitive impairment, neuropsychiatric symptoms, dysautonomia and sleep disturbance. ${ }^{1}$ Accurate diagnosis of PD is important both in clinical practice, where it will influence management, and in research, where the validity of findings may be compromised if studies include heterogenous conditions. This importance is likely to grow as neuroprotective strategies are developed that target specific pathological processes.

Unfortunately, accurate diagnosis of PD can be difficult. Definitive diagnosis can only be made at post mortem, by demonstration of depletion of brainstem pigmented neurones, with Lewy bodies in the remaining nerve cells. ${ }^{2}$ Antemortem diagnosis currently relies on clinical assessment but, in most post-mortem series, the positive predictive value (PPV) of the final clinical diagnosis of $\mathrm{PD}$ has only been found to be between $76 \%{ }^{3}$ and $90 \% .^{5}$ This can be improved with application of strict diagnostic criteria. For example, retrospective application of the UK Brain Bank diagnostic criteria ${ }^{3}$ improved the PPV in one study from $76 \%$ to $93 \%{ }^{6}$ However, application of these criteria meant that $32 \%$ of those with pathologically proven PD were not diagnosed clinically. Thus strict research based criteria increase specificity at the cost of reducing sensitivity.

Diagnosis at presentation or early in the disease course when the clinical picture may not yet have fully developed is even more problematic. Rajput and colleagues ${ }^{4}$ reported a PPV of $65 \%$ when comparing initial clinical diagnosis with pathological diagnosis, rising to $76 \%$ when comparing final clinical diagnosis with pathological diagnosis.

Much of the difficulty in the diagnosis of PD is in differentiating it from other disorders that cause parkinsonism. These include other neurodegenerative disorders such as dementia with Lewy bodies (DLB), progressive supranuclear palsy (PSP), multiple system atrophy and corticobasal degeneration, and non-degenerative causes such as vascular parkinsonism or drug induced parkinsonism (eg, as a result of antidopaminergic drugs). ${ }^{7}$ Difficulty in initial diagnosis is further compounded by the existence of non-parkinsonian disorders that can mimic parkinsonism. Particular problems are presented by tremor disorders, most notably essential tremor, and primary gait disorders, especially higher level disorders such as those associated with cerebrovascular disease. The increasing prevalence of multiple comorbidities with age can further complicate matters. Indeed, general slowing in the elderly can be confused with bradykinesia, with subtle extrapyramidal signs being reported in up to $35 \%$ of subjects older than 65 years. ${ }^{8}$

Although diagnosing the cause of parkinsonism is problematic, few studies have systematically assessed changes in the clinical diagnosis over time. One study found that in a cohort of patients with early presumed $\mathrm{PD}, 8 \%$ had their diagnosis changed after a mean follow-up of 6 years because of either a poor levodopa response, the development of atypical clinical features, atypical imaging findings or post mortem. ${ }^{9}$ However, this cohort is unlikely to be representative because the patients were part of a randomised clinical trial and the definition of atypical features was rather narrow.

We therefore set out to describe changes in diagnosis over time, factors associated with this change and the effect of application of strict research criteria on reaching a diagnosis in a 
Table 1 Diagnoses on clinical and research criteria at baseline and at the latest assessment

\begin{tabular}{llllc}
\hline & & & \multicolumn{2}{c}{ Latest diagnosis on research criteria } \\
\cline { 3 - 5 } & $\begin{array}{l}\text { Baseline clinical } \\
\text { diagnosis (n (\%)) }\end{array}$ & $\begin{array}{l}\text { Latest clinical } \\
\text { diagnosis (n (\%)) }\end{array}$ & All (n (\%)) & $\begin{array}{l}\text { Probable/definite } \\
\text { diagnoses only } \\
(\mathbf{n}=\mathbf{4 2})(\mathbf{n}(\%))\end{array}$ \\
\hline PD & $46(70)$ & $37(56)$ & $32(48.5)$ & $23(55)$ \\
DLB & $1(1.5)$ & $7(10.5)$ & $6(9)$ & $5(12)$ \\
Vascular & $5(7.5)$ & $9(14)$ & $10(15)$ & $8(19)$ \\
MSA & $4(6)$ & $3(4.5)$ & $5(7.5)$ & $4(9.5)$ \\
PSP & $1(1.5)$ & 0 & $2(3)$ & $2(4.5)$ \\
CBD & $1(1.5)$ & $1(1.5)$ & $1(1.5)$ & 0 \\
Other: parkinsonian/DIP & $3(4.5)$ & $2(3)$ & $3(5)^{*}$ & $\mathrm{~N} / \mathrm{A}$ \\
Other: non-parkinsonian & $5(7.5)$ & $7(10.5)$ & $7(10.5)^{*}$ & $\mathrm{~N} / \mathrm{A}$ \\
\hline
\end{tabular}

*Patients not meeting any applied research criteria. Parkinsonism defined as two or more of four cardinal motor signs.

CBD, corticobasal degeneration; DIP, drug induced parkinsonism; DLB, dementia with Lewy bodies; MSA, multiple system atrophy; PD, Parkinson's disease; PSP, progressive supranuclear palsy.

prospectively gathered cohort of incident patients who have undergone annual follow-up from initial diagnosis. The aims were to establish the proportion of patients who had their clinical diagnosis changed over time, the proportion who had their latest clinical diagnosis changed by the application of strict research criteria and the reasons for these changes.

\section{METHODS}

As part of the PINE pilot study, ${ }^{10}$ all patients from the lists of 18 general practices in Aberdeen (a population of approximately 148000 ) presenting with a newly diagnosed possible or definite parkinsonian syndrome (excluding definite drug induced parkinsonism) were recruited by multiple overlapping strategies over an 18 month period. All were seen by a consultant neurologist with a special interest in PD or a supervised trainee, and underwent a standardised baseline assessment, including the Unified Parkinson's Disease Rating Scale (UPDRS) ${ }^{11}$ with video recording of the motor section, and assessment of atypical features, such as dysautonomia, gaze palsy and cognitive or psychiatric symptoms. In those who consented to detailed follow-up, cognitive testing was carried out using the MiniMental State Examination (MMSE) ${ }^{12}$ and the Mini-Mental Parkinson (MMP), ${ }^{13}$ a PD specific cognitive screening tool. Consenting patients had repeat yearly assessments with planned lifelong follow-up, and most (59\%) were approached to give antemortem consent to post-mortem confirmation of the diagnosis.

Patients were asked to consent to undergo structural neuroimaging with either CT or MRI and functional imaging of dopamine transporter uptake with $\mathrm{N}$ - $\omega$-fluoropropyl-2 $\beta$ carbomethoxy-3 $\beta$-(4-iodophenyl)-tropane (FP-CIT) single photon emission computed tomography (SPECT). FP-CIT SPECT scans were visually graded by a blinded consultant

Table 2 Diagnostic changes

\begin{tabular}{lll}
\hline & $\begin{array}{l}\text { Between baseline } \\
\text { diagnosis and latest } \\
\text { clinical diagnosis } \\
(\mathbf{n}(\%))\end{array}$ & $\begin{array}{l}\text { Between latest } \\
\text { clinical diagnosis } \\
\text { and research } \\
\text { diagnosis (n (\%)) }\end{array}$ \\
\hline PD diagnosis unchanged & $33(50)$ & $32(48.5)$ \\
Other diagnosis unchanged & $11(16.5)$ & $26(39.5)$ \\
PD diagnosis changed to other diagnosis & $13(20)$ & $5(7.5)$ \\
$\begin{array}{l}\text { Other diagnosis changed to PD } \\
\text { Other diagnosis changed to other }\end{array}$ & $4(6)$ & $2(1.5)$ \\
diagnosis & $5(7.5)$ & $2(3)$
\end{tabular}

PD, Parkinson's disease. neuroradiologist as normal, abnormal (graded $1-3)^{14}$ or atypical (ie, abnormal but not in keeping with the pattern usually seen in neurodegenerative disease). ${ }^{15} \mathrm{CT}$ and MRI images were assessed visually for burden of cerebrovascular disease and, in the case of MRI, midbrain atrophy and basal ganglia signal change but no formal criteria were applied.

At baseline and at each yearly follow-up, the assessing (unblinded) clinician recorded up to three most likely clinical diagnoses without applying research criteria and gave a percentage certainty for each (eg, 90\% certain PD). Some patients were included in whom the most likely diagnosis was thought to be non-parkinsonian or drug induced parkinsonism, because it was thought that a degenerative or vascular parkinsonian syndrome remained a possibility. All available information was used to inform these diagnoses, including the results of any structural or functional imaging tests that were available at each assessment. Baseline diagnoses were reached after initial clinical assessment, before formal cognitive testing had been carried out.

On each patient's death, a final assessment was carried out, with review of general practitioner, hospital and research records, imaging tests and taking into account the results of post-mortem examinations where available. A final clinical or pathological diagnosis was then recorded.

In this study, 4 years after the beginning of the incident period, the initial and latest clinical diagnoses (the one with the highest percentage certainty) were compared for all incident patients who had at least 1 year of follow-up data and the reason for any change identified from the notes. In those patients who died, the final clinical diagnosis was taken from the yearly assessment immediately prior to death. Formal research criteria were then applied retrospectively using patients' research records at baseline and latest yearly followup. For 37 of the patients, these criteria were applied independently by two assessors and the diagnoses reached were compared to assess inter-rater reliability. Differences in diagnosis were resolved by discussion. The criteria applied were as follows: the UK Brain Bank criteria for $\mathrm{PD},{ }^{3}$ the consensus criteria for DLB, ${ }^{16}$ the consensus criteria for multiple system atrophy ${ }_{17}{ }^{17}$ the Litvan criteria for progressive supranuclear palsy ${ }_{18}^{18}$ Lees' proposed criteria for vascular parkinsonism ${ }^{19}$ and the Lang criteria for corticobasal degeneration ${ }^{20}$ disregarding cognitive decline as a criterion for exclusion. ${ }^{21}$ Where patients met more than one set of criteria, a single best fit diagnosis was decided upon by consensus of two authors based on the information available. 
Table 3 Baseline characteristics of those with and without a change in clinical diagnosis in all patients and in those initially diagnosed with Parkinson's disease

\begin{tabular}{|c|c|c|c|c|}
\hline & \multicolumn{2}{|l|}{ All patients } & \multicolumn{2}{|c|}{ Patients initially diagnosed with PD } \\
\hline & $\begin{array}{l}\text { No change in } \\
\text { diagnosis }(n=44)\end{array}$ & $\begin{array}{l}\text { Change in } \\
\text { diagnosis }(n=22)\end{array}$ & $\begin{array}{l}\text { No change in } \\
\text { diagnosis }(n=33)\end{array}$ & $\begin{array}{l}\text { Change in } \\
\text { diagnosis }(n=13)\end{array}$ \\
\hline Age (years) (mean (SD)) & $74.4(10.1)$ & $77.5(6.4)$ & $74.3(10.4)$ & $76.8(7.4)$ \\
\hline$\%$ Male & 54.5 & 77.3 & 48.5 & 76.9 \\
\hline Symptom duration (months) (median (IQR)) & $12.7(6.8-24.2)$ & $12.4(8.0-20.9)$ & $12.4(7.2-24.1)$ & $12.2(8.0-19.2)$ \\
\hline \multicolumn{5}{|l|}{ Symptoms described at baseline (n (\%)) } \\
\hline Tremor & $37(84.1)$ & $17(77.3)$ & $30(90.9)$ & $11(84.6)$ \\
\hline Bradykinesia & $31(70.5)$ & $15(68.2)$ & $24(72.7)$ & $10(76.9)$ \\
\hline Gait disturbance & $35(79.5)$ & $19(86.4)$ & $25(75.6)$ & $12(92.3)$ \\
\hline Postural instability & $23(52.3)$ & $14(63.6)$ & $15(45.5)$ & $9(69.2)$ \\
\hline MMSE (median (IQR)) & $28(26-29)$ & $25(24-28)$ & $28(26-29)$ & $27(24-28)$ \\
\hline MMP (median (IOR)) & $28(24-30)$ & $25(22-28)$ & $29(26-30)$ & $26(23-28)$ \\
\hline Motor UPDRS (mean (SD)) & $25.7(11.7)$ & $26.4(8.8)$ & $27.5(12.1)$ & $24.0(8.8)$ \\
\hline Total UPDRS (mean (SD)) & $37.9(17.3)$ & $38.9(14.0)$ & $39.5(18.7)$ & $35.5(12.5)$ \\
\hline
\end{tabular}

Rating Scale.

Statistical analysis was carried out using SPSS 14.0 for Windows. Median values were compared using the MannWhitney $U$ test, means using the Student's test and proportions using the $\chi^{2}$ test. Agreement was assessed using the kappa coefficient.

\section{RESULTS}

Of 82 incident patients identified, five were excluded from this study because they did not consent to follow-up and 11 were excluded because they died before their first yearly follow-up. The 66 remaining patients had a mean age of 75.0 (SD

Table 4 Reasons for change in clinical diagnosis

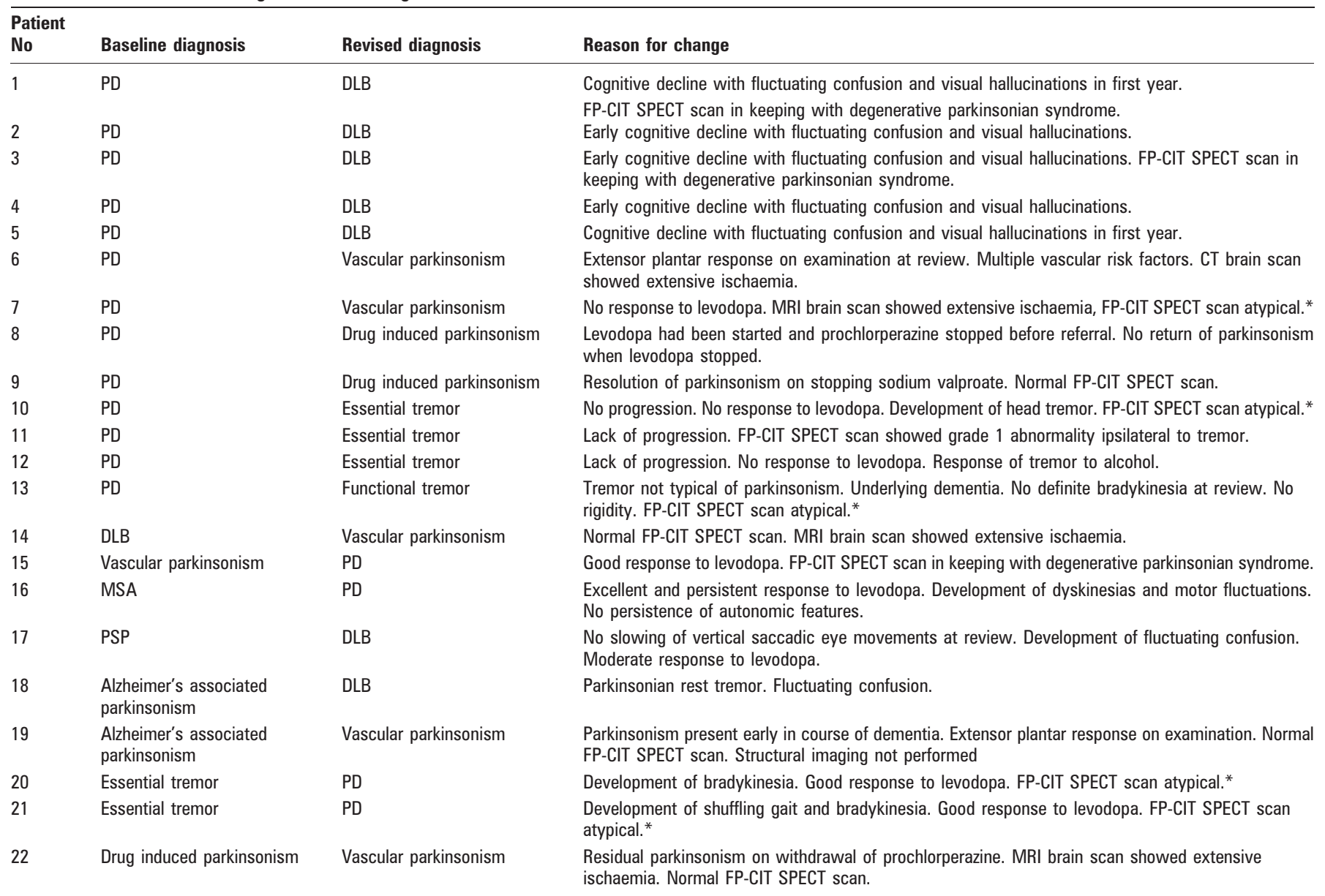

*FP-CIT scan showed punched out lesions not in keeping with the grading system used in degenerative parkinsonian syndromes. These were thought to be a result of cerebrovascular disease.

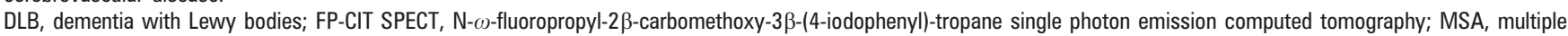
system atrophy; PD, Parkinson's disease; PSP, progressive supranuclear palsy. 
Table 5 Differences between latest clinical diagnosis and diagnosis on research criteria

\begin{tabular}{|c|c|c|c|}
\hline $\begin{array}{l}\text { Patient } \\
\text { No }\end{array}$ & Clinical diagnosis & $\begin{array}{l}\text { Diagnosis on clinical research } \\
\text { criteria or at post mortem }\end{array}$ & Notes \\
\hline 23 & $\begin{array}{l}\text { PD with coexistent } \\
\text { Alzheimer's disease }\end{array}$ & DLB & $\begin{array}{l}\text { Mild to moderate dementia developing } 3 \text { y before onset of asymmetric parkinsonism with tremor. } \\
\text { Dementia diagnosed as Alzheimer's disease by psychiatrists. No fluctuating confusion or } \\
\text { hallucinations. }\end{array}$ \\
\hline 24 & $\mathrm{PD}$ & MSA & $\begin{array}{l}\text { Asymmetric bradykinesia and rest tremor. Yet to undergo full trial of dopaminergic therapy. } \\
\text { Otherwise unexplained urinary urgency and incontinence. }\end{array}$ \\
\hline 25 & PD & MSA & $\begin{array}{l}\text { Asymmetric parkinsonism, dominated by tremor. Postural hypotension prior to dopaminergic } \\
\text { therapy. Mediocre response to levodopa. }\end{array}$ \\
\hline 26 & PD & $\mathrm{PSP}^{*}$ & $\begin{array}{l}\text { Typical asymmetric rest tremor. Poor levodopa response. No gaze palsy. Early balance problems, } \\
\text { attributed to hip fracture. Post-mortem diagnosis of progressive supranuclear palsy. }\end{array}$ \\
\hline 27 & PD & $\mathrm{PSP}^{*}$ & $\begin{array}{l}\text { Asymmetric parkinsonism with tremor. No gaze palsy. Poor objective response to levodopa, but } \\
\text { assessment made difficult by previous stroke. Early balance problems, attributed to stroke. Post- } \\
\text { mortem diagnosis of progressive supranuclear palsy. }\end{array}$ \\
\hline 28 & PD & Parkinsonism, unspecified & $\begin{array}{l}\text { Unilateral parkinsonism with rest tremor, remaining unilateral after } 4 \mathrm{y} \text { of follow-up. FP-CIT in } \\
\text { keeping with degenerative parkinsonian syndrome. MRI brain scan normal. }\end{array}$ \\
\hline 29 & DLB & PD & $\begin{array}{l}\text { First onset of symptoms in 2001. Onset of dementia with fluctuating confusion and hallucinations in } \\
2004 \text {, quickly coming to dominate clinical picture. }\end{array}$ \\
\hline 30 & DLB & Vascular parkinsonism & $\begin{array}{l}\text { Asymmetric bradykinesia and rest tremor affecting face and left hand. Dementia with fluctuations in } \\
\text { confusion but no hallucinations. MRI brain showed extensive ischaemia. FP-CIT SPECT scan } \\
\text { atypical. }\end{array}$ \\
\hline
\end{tabular}

*Did not meet research diagnostic criteria prior to post mortem.

DLB, dementia with Lewy bodies; FP-CIT SPECT, N- $\omega$-fluoropropyl-2 $\beta$-carbomethoxy-3 $\beta$-(4-iodophenyl)-tropane single photon emission computed tomography; MSA, multiple system atrophy; PD, Parkinson's disease; PSP, progressive supranuclear palsy.

$10.5)$ years at diagnosis and were predominantly male $(n=41$ $(62 \%))$. At the initial assessment, symptoms had been present for a median of 12.5 months (interquartile range (IOR) 8 to 24). Twenty-four patients died after at least 1 year of follow-up but no other patients were lost to follow-up. Only five patients had undergone examination of the brain at post mortem.

\section{Change in clinical diagnosis}

Forty-six patients (70\%) were initially diagnosed with idiopathic $\mathrm{PD}$, with the most common alternate diagnoses being vascular parkinsonism, multiple system atrophy (all parkinsonian variant) and essential tremor (diagnosed in three patients; table 1). After a median follow-up of 29 months (IOR 23 to 33), the number diagnosed clinically with PD had fallen to $37(56 \%)$, the most common alternative diagnoses being vascular parkinsonism and DLB.

The clinical diagnosis changed between the baseline assessment and the latest follow-up in 22 patients (33\%) and remained unchanged in 44 (table 2). There was no significant difference between these two groups in presenting symptoms, severity of their parkinsonian impairment (UPDRS) or duration of their symptoms at baseline (table 3). There was a nonsignificant trend towards greater cognitive impairment (MMSE and $\mathrm{MMP}$ ) and older age in the group whose diagnoses changed. Most $(18,82 \%)$ changes occurred in the first year, three $(6 \%$ of those with at least 2 years of follow-up) occurred within the second and one ( $3 \%$ of those with at least 3 years of follow-up) within the third.

In those initially diagnosed with $\mathrm{PD}$, the diagnosis was most likely to change to DLB $(5 / 13,38 \%)$ or essential tremor $(3 / 13$, $23 \%$ ) (table 4). Changes were most commonly a result of development of additional features $(n=7)$, particularly early cognitive impairment and neuropsychiatric features $(n=5)$, the results of radiological imaging $(n=6)$, poor response to levodopa $(n=4)$ and lack of disease progression $(n=6)$.

Changes in diagnosis in those initially diagnosed with conditions other than $\mathrm{PD}$ are shown in table 4 . Changes were to $\mathrm{PD}(4 / 9,44 \%)$, vascular parkinsonism $(3 / 9,33 \%)$ or $\mathrm{DLB}(2 / 9$, $22 \%)$. The predominant reason for change to a diagnosis of $\mathrm{PD}$ was response to levodopa. In the two patients whose diagnoses changed from essential tremor to PD, both had FP-CIT SPECT scans that were abnormal in a pattern not in keeping with the usual pattern in neurodegenerative disorders and were reported as being likely to be indicative of cerebrovascular disease. Clinically, these patients were not felt likely to suffer from vascular parkinsonism. All three patients whose diagnoses changed to vascular parkinsonism had normal FP-CIT SPECT scans.

\section{Differences between latest clinical diagnosis and research diagnosis}

Agreement between the two observers on the research criteria diagnosis was good $(\kappa=0.73)$, and agreement between research diagnosis and clinical diagnosis was very good $(\kappa=0.82)$. Details of the eight participants whose clinical and research diagnoses differed are shown in table 5.

When research criteria were applied using only the information available at baseline, 46 patients had a "possible" diagnosis according to the criteria, 10 had a "probable" diagnosis and 10 could not be diagnosed using the criteria applied. Where the most up to date information was used, 10 patients could not be diagnosed (three had an unspecified parkinsonian syndrome and seven were not parkinsonian), 14 had a "possible" diagnosis, 37 had a "probable" diagnosis and five had a "definite" diagnosis (table 1). Of the 14 patients whose diagnosis by research criteria was rated "possible", six (43\%) also met the criteria for "possible" diagnosis of another syndrome.

In the five patients who underwent post-mortem examination, the final clinical, research and pathological diagnoses agreed in two (one PD and one vascular) and in one the clinical diagnosis and pathological diagnosis agreed (PD with coexistent Alzheimer's disease) while research criteria suggested possible DLB. The two participants whose clinical and antemortem research diagnoses were $\mathrm{PD}$ but whose post-mortem examinations showed PSP are described in table 5.

\section{DISCUSSION}

In this study, one-third of the initial diagnoses of the cause of parkinsonism changed over a median of 29 months of followup. The majority of this change was a result of initial 
overdiagnosis of PD. The proportion of patients diagnosed with PD fell by $14 \%$ and most (59\%) diagnostic changes were away from PD. These results are similar to previous studies, which showed that the clinical diagnosis of parkinsonism changed over time in $36 \%$ of patients attending a highly specialist clinic, ${ }^{22}$ and $16 \%$ of an incident cohort of patients with PD according to UK Brain Bank criteria had their initial diagnosis changed after about 3.5 years of follow-up. ${ }^{23}$

Over one-third (38\%) of those misdiagnosed with PD had their diagnosis changed to DLB. This was usually because the symptomatic cognitive features of the disease were absent initially as, even using the strictest interpretation of the diagnostic criteria, parkinsonism can predate the onset of dementia by up to 1 year. ${ }^{16}$ However, the suggestion of a difference in cognitive scores between the change and no change groups would suggest that there may have been detectable deficits despite the lack of cognitive symptoms. The MMSE and MMP may, therefore, be of some use in identifying parkinsonian patients who will go on to develop early dementia, although a larger study is needed to test this hypothesis.

Essential tremor was clinically misdiagnosed as PD in three of our patients, which is not unexpected. While an essential tremor is characteristically more prominent on posture holding and action, it can also cause a tremor at rest ${ }^{24}$ and may even be associated with rigidity. ${ }^{25}$ Conversely, PD may present with an isolated asymmetric postural tremor in the absence of other features and may only cause other features of parkinsonism after many years. ${ }^{26}$

Changes in diagnosis were usually as a result of level of response to treatment with levodopa or a dopamine agonist, lack of progression and development or resolution of atypical clinical features. This serves to highlight the need for regular follow-up and diagnostic revision in parkinsonian patients, and the diagnostic value of a trial of therapy. However, the results of therapeutic trials must be interpreted with caution, as PD may not show the classical excellent response to treatment, ${ }^{27}$ and there have been reports of pathologically established cases of PD without an appreciable response to levodopa ${ }^{28}$ making clinical diagnosis in life extremely difficult. The role of functional imaging in the changes to diagnosis that took place is unclear. The results of FP-CIT SPECT scans supported change from initial clinical diagnoses in six patients, although in all but one of these cases (patient No 14, table 4), the clinical features were present that may have led to the change independently. In the two changes in diagnosis from essential tremor to $\mathrm{PD}$, the FP-CIT SPECT scan results were not typical of a neurodegenerative disorder.

The latest clinical diagnosis differed from the diagnosis on research criteria in eight patients $(12 \%$; table 5$)$ usually because of exclusion criteria within the research criteria (patient Nos 23 and 24), or arbitrary time limits within the criteria (patient Nos 28 and 29). In two cases (patient Nos 25 and 30) there were features to support both diagnoses and it could be argued that either could be applied. In two patients (patient Nos 26 and 27) whose pathological diagnosis differed from their research and latest clinical diagnosis, PSP was mistaken for PD. It is increasingly recognised that, as well as causing the typical syndrome of postural instability, supranuclear gaze palsy and cognitive dysfunction (Richardson's syndrome), PSP can present with an asymmetric parkinsonian syndrome very similar to PD, although with a blunted response to dopaminergic therapy (PSP parkinsonism). ${ }^{29}$

Research criteria were of limited value in supporting a clinical diagnosis in parkinsonian patients. At baseline assessment, most
$(70 \%)$ diagnoses were rated "possible" and only 10 patients $(15 \%)$ met the criteria for "probable" diagnosis of a parkinsonian syndrome. Even after about 2.5 years of follow-up, a significant proportion of diagnoses in those with a parkinsonian disorder remained unclassifiable (5\%) or "possible" $(24 \%)$ and nearly half of the latter met criteria for another "possible" diagnosis.

The main strength of our study was that it involved a community based incident cohort where steps were taken to identify as high a proportion of parkinsonian patients as possible, standardised prospective assessments were made each year by a specialist with an interest in movement disorders and few patients were lost to follow-up. The patients are, therefore, likely to be representative of parkinsonian patients in general, and not only those usually seen at specialist clinics, while the prospective data collection allowed accurate application of the research criteria.

However, there are also some limitations of this study that are worth noting. Firstly, our cohort was relatively small as it was drawn from a pilot study. A larger incidence study is currently underway and will allow analysis of similar data in a larger population. Secondly, while the revised diagnoses here are taken to be correct, it is likely that they will continue to change over time. Follow-up of this cohort will continue and so it will be possible to report the pattern of changes with longer followup in the future. Thirdly, the diagnoses were all supervised by a single consultant with an interest in PD and so our results may not be generalisable, particularly to more generalist clinics where the misdiagnosis rate may be higher. Finally and importantly, few diagnoses have been confirmed by post mortem and, therefore, it is not clear whether either the clinical or antemortem research diagnoses are correct. This is a problem with all clinical studies of parkinsonism but, because we have systematically tried to approach our participants for antemortem consent, we hope to obtain pathological confirmation in more of our participants over time. Studies of diagnostic accuracy that used brain bank material ${ }^{3-5}$ had the advantage of complete pathological confirmation but were disadvantaged by limited clinical information on which to base research diagnostic criteria and limited generalisability because post mortems are often performed on unusual or difficult cases.

The clinical significance of misdiagnoses in parkinsonism varies. A change in diagnosis between a parkinsonian condition and a non-parkinsonian one (such as essential tremor) will have a significant impact on patient care as the treatments are quite different. Changing between different parkinsonian syndromes may not alter management so dramatically as a trial of dopamine replacement therapy is often warranted but it will alter what information is given to the patient about prognosis. In addition, some clinicians may wish to avoid early levodopa in those with PD because of the risk of motor complications, while treatment withdrawal should always be considered where the syndrome is thought to be unresponsive. Similarly, while many experts would regard PD and DLB as being part of the same disease spectrum, we regarded them separately because their prognosis differs and dopamine agonists may be less suitable in DLB because of their greater neuropsychiatric toxicity.

In summary, we have demonstrated that even in those with a special interest, the accurate diagnosis of the cause of parkinsonism at presentation was difficult, that $\mathrm{PD}$ was overdiagnosed at first assessment and that research criteria were often unhelpful in clarifying the diagnosis, even after a median of 29 months of follow-up. Further research, in larger groups and over longer periods, is necessary to identify factors 
that may be used to improve the accuracy of diagnosis at the initial assessment. Our findings support the recent NICE guidelines that regular clinical review of those suffering from parkinsonism with careful attention to the diagnosis is essential in order that they receive appropriate care. ${ }^{30}$

Funding: RC is supported by a grant from the Parkinson's Disease Society.

Competing interests: None.

Ethics approval: Obtained.

\section{REFERENCES}

1. Chaudhuri KR, Healy DG, Schapira AH. Non-motor symptoms of Parkinson's disease: diagnosis and management. Lancet Neurol 2006;5:235-45.

2. Oppenheimer DR, Esiri MM. Diseases of the basal ganglia, cerebellum and motor neurons. In: Adams JH, Duchen LW, eds. Greenfield's neuropathology, 5th Edn. London: Edward Arnold; 1992:988-1045.

3. Hughes AJ, Daniel SE, Kilford L, et al. Accuracy of clinical diagnosis of idiopathic Parkinson's disease: a clinico-pathological study of 100 cases. J Neurol Neurosurg Psychiatry 1992:55:181-4.

4. Rajput AH, Rozdilsky B, Rajput A. Accuracy of clinical diagnosis in parkinsonism-a prospective study. Can J Neurol Sci 1991:18:275-8.

5. Hughes AJ, Daniel SE, Lees AJ. Improved accuracy of clinical diagnosis of Lewy body Parkinson's disease. Neurology 2001;57:1497-9.

6. Hughes AJ, Ben-Shlomo Y, Daniel SE, et al. What features improve the accuracy of clinical diagnosis in Parkinson's disease: A clinicopathologic study. Neurology 1992;42:1142-6.

7. Taylor KSM, Counsell C. Is it Parkinson's disease, and if not, what is it? Pract Neurol 2006:6:154-65.

8. Bennett DA, Beckett LA, Murray AM, et al. Prevalence of parkinsonian signs and associated mortality in a community population of older people. N Engl J Med 1996:334:71-6.

9. Jankovic J, Rajput AH, McDermott MP, et al. The evolution of diagnosis in early Parkinson disease. Arch Neurol 2000:57:369-72.

10. Taylor KS, Counsell CE, Harris CE, et al. Pilot study of the incidence and prognosis of degenerative parkinsonian disorders in Aberdeen, United Kingdom: methods and preliminary results. Mov Disord 2006:21:976-82.

11. Fahn S, Elton RL, UPDRS Development Committee. The Unified Parkinson's Disease Rating Scale. In: Fahn S, Marsden CD, Calne D, et al, eds. Recent developments in Parkinson's disease, vol 2. Florham Park, New Jersey: Macmillan Healthcare Information 1987:153-63.

12. Folstein MF, Folstein SE, McHugh PR. "Mini-mental state". A practical method for grading the cognitive state of patients for the clinician. J Psychiatr Res 1975;12:189-98.

13. Mahieux F, Michelet D, Manifacier M-J, et al. Mini-Mental Parkinson: First validation study of a new bedside test constructed for Parkinson's disease. Behav Neurol 1995;8:15-22.
14. Benamer HTS, Patterson J, Grosset DG, et al. Accurate differentiation of parkinsonism and essential tremor using visual assessment of [123I]-FP-CIT SPECT imaging: The [123I]-FP-CIT study group. Mov Disord 2000:15:503-10.

15. Marshall V, Grosset DG. Role of dopamine transporter imaging in routine clinical practice. Mov Disord 2003;18:1415-23.

16. McKeith IG, Dickson DW, Lowe JDM, et al. Diagnosis and management of dementia with Lewy bodies: Third report of the DLB consortium. Neurology 2005; 65:1863-72.

17. Gilman S, Low PA, Quinn $\mathrm{N}$, et al. Consensus statement on the diagnosis of multiple system atrophy. J Neurol Sci 1999;163:94-8.

18. Litvan I, Agid Y, Calne D, et al. Clinical research criteria for the diagnosis of progressive supranuclear palsy (Steele-Richardson-Olszewski syndrome): Report of the NINDS-SPSP International Workshop. Neurology 1996;47:1-9.

19. Zijlmans JC, Daniel SE, Hughes AJ, et al. Clinicopathological investigation of vascular parkinsonism, including clinical criteria for diagnosis. Mov Disord 2004: 19:630-40.

20. Lang AE, Riley DE, Bergeron C. Cortical-basal ganglionic degeneration. In: Calne DB, ed. Neurodegenerative diseases. Philadelphia: WB Saunders, 1994:877-94.

21. Riley DE, Lang AE. Clinical diagnostic criteria. In: Litvan I, Goetz CG, Lang AE, eds. Corticobasal degeneration and related disorders. Philadelphia: WB Saunders; 2000:29-34.

22. Hughes AJ, Daniel SE, Ben-Shlomo Y, et al. The accuracy of diagnosis of parkinsonian syndromes in a specialist movement disorder service. Brain 2002:125:861-70.

23. Williams-Gray $\mathbf{C H}$, Foltynie T, Brayne CEG, et al. Evolution of cognitive dysfunction in an incident Parkinson's disease cohort. Brain 2007:130:1787-98.

24. Cohen 0, Pullman S, Jurewicz E, et al. Rest tremor in patients with essential tremor: prevalence, clinical correlates, and electrophysiologic characteristics. Arch Neurol 2003;60:405-10.

25. Deuschl G, Bain P, Brin M. Consensus statement of the Movement Disorder Society on Tremor. Mov Disord 1998;13(Suppl 3):2-23.

26. Chaudhuri KR, Buxton-Thomas M, Dhawan V, et al. Long duration asymmetrical postural tremor is likely to predict development of Parkinson's disease and not essential tremor: Clinical follow up study of 13 cases. J Neurol Neurosurg Psychiatry 2005;76:115-17.

27. Wenning GK, Ben-Shlomo Y, Hughes A, et al. What clinical features are most useful to distinguish definite multiple system atrophy from Parkinson's disease? J Neurol Neurosurg Psychiatry 2000;68:434-40.

28. Mark MH, Sage JI, Dickson DW, et al. Levodopa-nonresponsive Lewy body parkinsonism: clinicopathologic study of two cases. Neurology 1992:42:1323-7.

29. Williams DR, De Silva R, Paviour DC, et al. Characteristics of two distinct clinical phenotypes in pathologically proven progressive supranuclear palsy: Richardson's syndrome and PSP-parkinsonism. Brain 2005;128:1247-58.

30. National Collaborating Centre for Chronic Conditions. Parkinson's disease: national clinical guideline for diagnosis and management in primary and secondary care. London: Royal College of Physicians, 2006. 